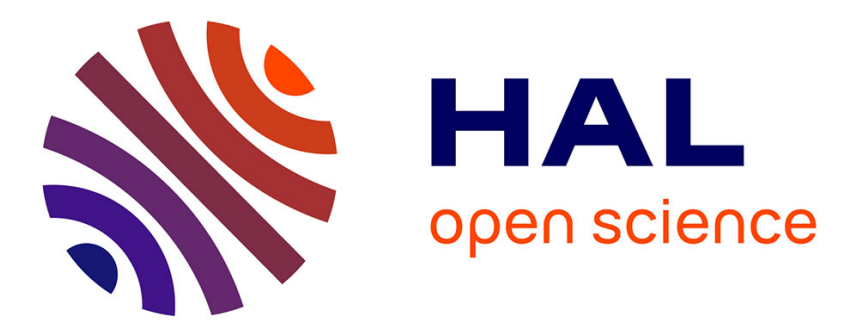

\title{
Computer investigations of the influences of magnets for magnetron-sputtering
}

\author{
O. Knotek, F. Löffler, U. Schnaut, W. Guan
}

\section{To cite this version:}

O. Knotek, F. Löffler, U. Schnaut, W. Guan. Computer investigations of the influences of magnets for magnetron-sputtering. Journal de Physique IV Proceedings, 1993, 03 (C7), pp.C7-1211-C7-1216. 10.1051/jp4:19937186 . jpa-00251818

\section{HAL Id: jpa-00251818 https://hal.science/jpa-00251818}

Submitted on 1 Jan 1993

HAL is a multi-disciplinary open access archive for the deposit and dissemination of scientific research documents, whether they are published or not. The documents may come from teaching and research institutions in France or abroad, or from public or private research centers.
L'archive ouverte pluridisciplinaire HAL, est destinée au dépôt et à la diffusion de documents scientifiques de niveau recherche, publiés ou non, émanant des établissements d'enseignement et de recherche français ou étrangers, des laboratoires publics ou privés. 


\title{
Computer investigations of the influences of magnets for magnetron- sputtering
}

\author{
O. KNOTEK, F. LÖFFLER, U. SCHNAUT and W. GUAN
}

Institut für Werkstoffkunde (IWKB), Aachen University of Technology, Templergraben 55, 5100 Aachen, Germany

\section{Introduction}

For a long time PVD (Physical-Vapour-Deposition) technology has been applied in various modern technical fields, owing to the wide range of possible coating materials whose potential is virtually unlimited. Besides electronic, optical and decorative applications for PVD films, hard films, in particular, have been widely applied for tool coatings which play important roles for the resistance against wear and corrosion $/ 1,2 \%$. The MSIP (Magnetron-Sputter-Ion-Plating) coating process is one of the PVDprocesses, providing 1) relatively high deposition rates, 2) large deposition areas, and 3) low substrate heating $/ 3 /$. The magnetron behind the target influences the distribution of the electrons moving in the plasma and the space between the cathode and anode, thus the distribution of the ions impinging on the surface of the target. Since the electrons are distributed unevenly in front of the target, the working gas atoms are therefore unevenly ionized in the plasma and the ions also unevenly bombard the target surface and the erosion of the target surface is uneven. Therefore the investigations of the magnetic field in the MSIP are very important for the optimization of the deposition process and the usage of the target. Generally, the accurate calculations or measurements of the direction and strength of the magnetic field (magnetic induction or magnetic flux density $\widetilde{\mathbf{B}}$ ) is difficult in comparison to measurements of the electrical field, because $\overrightarrow{\mathbf{B}}$ is a vector with varying direction and magnitude.

In this paper, it is shown that a magnetical field of a cylindrical magnetron in MSIP-equipment can be quickly simulated through a simple model and the distribution of the field is discussed.

\section{Arrangement of a typical magnetron}

By reviewing the limitation of the conventional planar diodes sputtering, one can clearly see the advantage of the MSIP. Fig. 1 shows the principles of both processes. In the diode sputtering the sputter rate of the target is very low and therefore the deposition rate of the coatings is also low because in this process the ionization rate of the working gas (generally Ar) is low /1/. In order to increase the ionization of the working gas a magnetron is installed behind the target and a drift field $\overrightarrow{\mathbf{E}} \times \overrightarrow{\mathbf{B}}$ is formed in the front of the target surface if an electric field between the cathode and anode is present.

The electron motion in the drift field $\overrightarrow{\mathbf{E}} \times \overrightarrow{\mathbf{B}}$ can be understood as follows: When an electron is in an uniform magnetic field, its motion perpendicular to the field lines can be pictured as an orbit around a field line. Its motion along the field is unimpeded, so that if it has a component of velocity along the field 
line, its net motion is a spiral along the field line. Thus such electrons can be considered to be trapped on magnetic field lines. An electron trapped on a given field line can advance to an adjacent field line by suffering a collision. An electron will undergo a drift motion across the magnetic field. The direction of the electron motion in the drift field $\overline{\mathbf{E}} \times \overline{\mathbf{B}}$ i.e. the direction of the drift field $\overline{\mathbf{E}} \times \overline{\mathbf{B}}$, is not the same as that of the electric field, but in a direction perpendicular to both the electric and magnetic fields. Thus a drift current in the drift field $\overrightarrow{\mathbf{E}} \times \overrightarrow{\mathbf{B}}$ is formed. The electrons which are in the drift current collide with the working gas atoms/molecules and then the possibility of ionizations of the working gas atoms/molecules is increased. A typical magnetron used in the MSIP-process is represented in Fig. 1(a) /1/. This magnetron consists of four parts - a cathode (target), a cylindrical magnet, a circular magnet or soft iron and a round soft iron as pole piece.

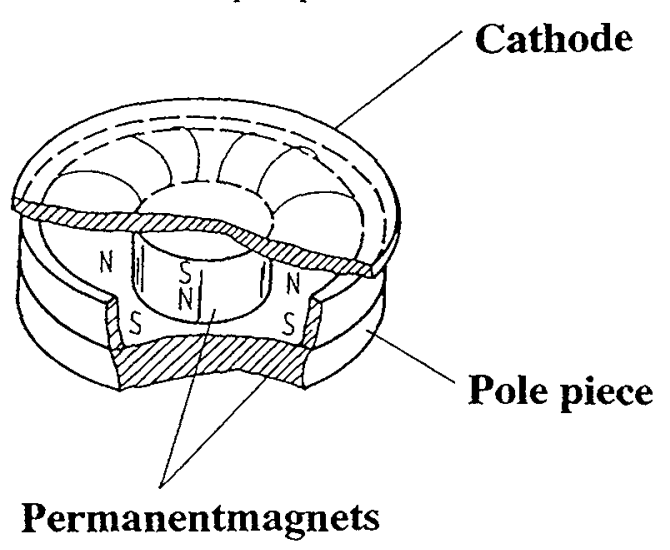

(a)

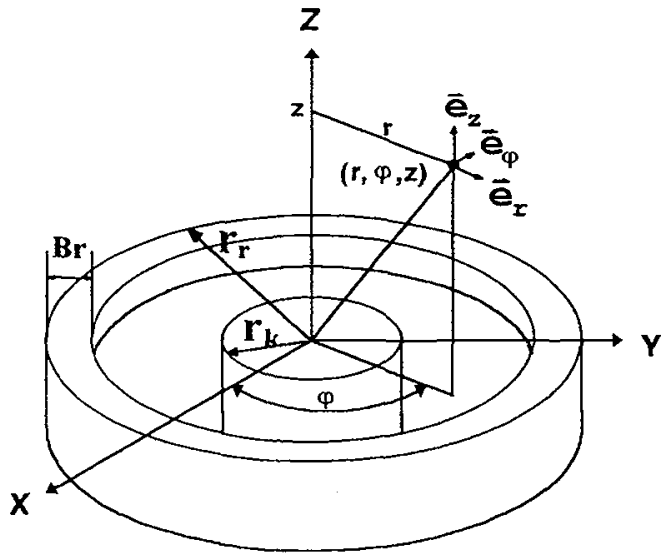

(b)

Fig. 1: Schematic illustrations of a typical cylindrical magnetron /1/ and its geometry and symmetry

\section{A simple model}

The analyses of an electromagnetic field start from the Maxwell equations /4/. The differential Maxwell equations are:

$$
\begin{aligned}
& \operatorname{rot} \overline{\mathbf{H}}=\overrightarrow{\mathbf{J}}+\frac{\partial \overline{\mathbf{D}}}{\partial \mathrm{t}} \\
& \operatorname{rot} \overline{\mathbf{E}}=-\frac{\partial \overline{\mathbf{B}}}{\partial \mathrm{t}} \\
& \operatorname{div} \overline{\mathbf{B}}=\rho \\
& \operatorname{div} \overline{\mathbf{D}}=0
\end{aligned}
$$

with: $\overrightarrow{\mathbf{H}}$ : magnetic field $[\mathrm{A} / \mathrm{m}]$

$\overrightarrow{\mathbf{J}}$ : current density $\left[\mathrm{A} / \mathrm{m}^{2}\right]$

$\overrightarrow{\mathbf{B}}:$ magnetic induction $\left[\mathrm{Vs} / \mathrm{m}^{2}\right], \quad \overline{\mathbf{E}}:$ electric field $[\mathrm{V} / \mathrm{m}]$

$\rho$ : charge density $\left[\mathrm{As} / \mathrm{m}^{3}\right], \quad \overline{\mathbf{D}}$ : displacement $\left[\mathrm{As} / \mathrm{m}^{2}\right]$

In the linear and isotopic medium there are linear relationships between the field strength $\overrightarrow{\mathbf{H}}$ and $\overrightarrow{\mathbf{E}}$ with the magnetic induction $\overline{\mathbf{B}}$ and displacement $\overline{\mathbf{D}}$ :

$$
\begin{aligned}
& \overrightarrow{\mathbf{B}}=\mu \overrightarrow{\mathbf{H}} \\
& \overrightarrow{\mathbf{D}}=\varepsilon \overrightarrow{\mathbf{E}}
\end{aligned}
$$

Investigating the electromagnetic field with the magnetron showed in Fig. 1(a), the following assumptions are made:

- The electromagnetic field system has a cylindrical symmetry and thus a cylindrical coordinate is 
arranged, shown in Fig. 1(b).

- Only the deposition processes with DC-mode are considered, i.e. $\frac{\partial \overrightarrow{\mathbf{D}}}{\partial \mathrm{t}}=0$ and $\frac{\partial \overrightarrow{\mathbf{B}}}{\partial \mathrm{t}}=0$ in the Maxwell equations.

- The current in the deposition process flows along the axis of the process space to the cathode and its density at any position depends only on the distance from the axis $r$ i.e. $\overrightarrow{\mathbf{J}}=j_{\mathrm{Ar}^{r}}(r) \overrightarrow{\mathbf{e}}_{\mathrm{z}}$ in the Maxwell equations,

- The influences of boundary effects of other elements in the process space are neglected and it is assumed that the medium in the space is vacuum, i.e. the formulas (5) and (6) are available with $\mu=\mu_{0}=4 \pi \cdot 10^{-7}[\mathrm{Vs} / \mathrm{Am}]$ and $\varepsilon=\varepsilon_{0}=8.8542 \cdot 10^{-12}[\mathrm{As} / \mathrm{Vm}]$. The magnetic field can be demonstrated by field strength $\overrightarrow{\mathbf{H}}$ or induction $\overrightarrow{\mathbf{B}}$.

On the basis of these assumptions the magnetic field in the process space with the cylindrical system can be represented by Bessel functions through the solutions of the Maxwell equations with separation of the variables:

$$
\begin{aligned}
& H_{r}(r, z)=H_{m r} e^{-\lambda_{r} \cdot z} J_{1}\left(\lambda_{r} \cdot r\right) \\
& H_{z}(r, z)=H_{m z} e^{-\lambda_{z} \cdot z} J_{0}\left(\lambda_{z} \cdot r\right) \\
& H_{\varphi}(r, z)=\frac{1}{r} \int_{0}^{r} r \cdot j_{A r^{+}}(r) \cdot d r
\end{aligned}
$$

where $\mathrm{H}_{\mathrm{mr}}$ and $\mathrm{H}_{\mathrm{mz}}$ are the maximum of the components of the magnetic field at the target surface $(\mathrm{Z}=0), \mathrm{J}_{0}, \mathrm{~J}_{1}$ are the Bessel functions, and the decline factors $\lambda_{\mathrm{r}}$ and $\lambda_{\mathrm{z}}$ are determined from the dimen-sions of the magnetron (Fig. 1(b)) and the properties of the Bessel functions.

In real processes it has been observed that at the target surface the maximum of the r-component and the minimum of the $z$-component of the magnetic field appear at the same positions $r=r_{0}$. From that one can obtain:

$$
\begin{aligned}
& \lambda_{\mathrm{r}}=\frac{1.8412}{\mathrm{r}_{0}} \\
& \lambda_{\mathrm{z}}=\frac{2.4048}{\mathrm{r}_{0}} \\
& \mathrm{r}_{0}=\frac{\mathrm{r}_{\mathrm{r}}+\mathrm{r}_{\mathrm{k}}-\mathrm{B}_{\mathrm{r}}}{2}
\end{aligned}
$$

These geometrical dimensions for a magnetron in the MSIP equipment used in this study are following: $\mathrm{r}_{\mathrm{k}}=15[\mathrm{~mm}], \mathrm{r}_{\mathrm{r}}=35[\mathrm{~mm}], \mathrm{B}_{\mathrm{r}}=8[\mathrm{~mm}]$. In the present paper two types of magnetrons with various combinations of magnetic elements are considered:

Type I: central cylindrical magnet with circular magnet

Type II: central cylindrical magnet with circular soft iron

Under the consideration of the various types of the magnetron two corrective factors $\beta_{\mathrm{r}}$ and $\beta_{\mathrm{z}}$ are obtained from the measured magnetic field: 
for type I: $\begin{array}{ll}\beta_{\mathrm{r}}=2.2469 \\ \beta_{\mathrm{z}}=1.7543\end{array}$ for type II: $\beta_{\mathrm{r}}=3.0171$

$\beta_{\mathrm{Z}}=2.2675$

The experimental measurements of the magnetic field was carried out in air and under static state, e.g. $\mathrm{j}_{\mathrm{Ar}^{+}}(\mathrm{r})=0$. Thus the magnetic field at any position in the process space can be written as follows:

$$
\begin{aligned}
& H_{r}(r, z)=H_{m r} e^{-\lambda_{r} \beta_{r} \cdot z} J_{1}\left(\lambda_{r} \cdot r\right) \\
& H_{z}(r, z)=H_{m z} e^{-\lambda_{2} \beta_{z} \cdot z} J_{0}\left(\lambda_{z} \cdot r\right)
\end{aligned}
$$

\section{Results from calculations and measurements}

Fig. 2 shows the distributions of magnetic induction at the target surface $(z=0)$ from measurements through a Hall instrument and the simulations using the equ. (10) and (11), whose maxima $\mathrm{H}_{\mathrm{mr}}$ and $\mathrm{H}_{\mathrm{mz}}$ are obtained by measurements. These distributions can be seen as boundary values of the magnetic field in the MSIP process space. From Fig. 2 one can see that this boundary values of the magnetic field can be well described by equ. (10) and (11) if the maxima of the components of the field are known. Only at the edge positions of the target the relative large differences appear due to the edge effects in the measurements and the neglection of these effects in the model.

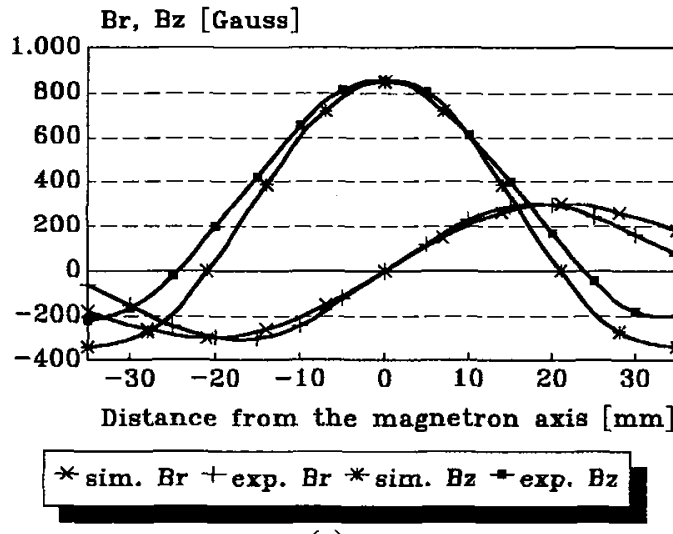

(a)

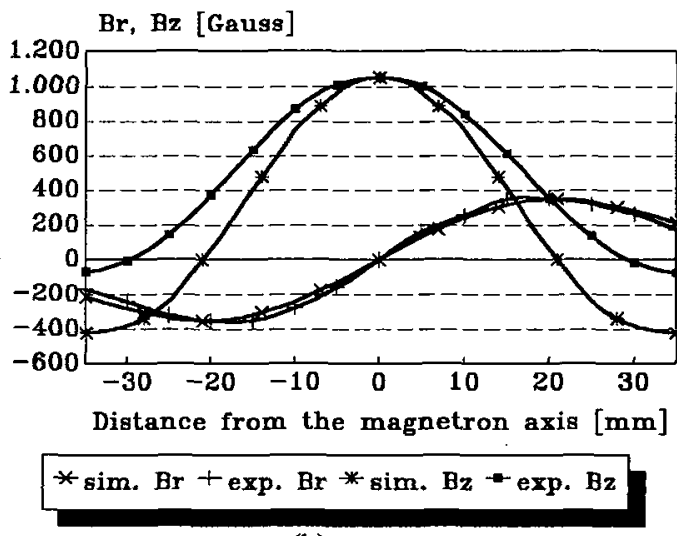

(b)

Fig. 2: The measured and calculated boundary values of the magnetic field at the target surface $(\mathrm{z}=0 \mathrm{~mm})$ for the different magnetron types (a): type I, (b): type II

The substrates are placed at the positions with a distance of $20 \mathrm{~mm}$ to the target surface. The magnetic field distributions at the surface of the substrate are illustrated in Fig. 3. The results of the calculations show good agreement with measurements. The distributions show similar behaviour with the distributions of the boundary values.

In order to research the declines of the magnetic field in the process space of various magnetron types one assumes that the magnetrons have the same maximum of the boundary values at the target surface and calculates the magnetic field at the positions at $r=0$ (for $B_{-} z$ ) and $r=r_{0}$ (for $B_{r} r$ ) along the $z-$ direction. In Fig. 4 the results show that the decline in magnetron I is larger than that in magnetron II. This is caused by the relative large part of magnetic flux led by the magnetic circuit in the magnetron I which consists of cylindrical and circular magnets.

The distributions of the magnetic field in the process space are calculated by the equ. (10) and (11) and 
represented in Fig. 5 where $B r, B z$ and $B$ rz represent correspondingly the components of the magnetic field in $r$ and $z$ directions and the absolute value of the field. From Fig. 5 one can find that the distribution of $B_{-} r$ is similar to the contour of the erosion zone of the target. It means that $B_{-} r$ has direct influence on the sputtering process at the target.

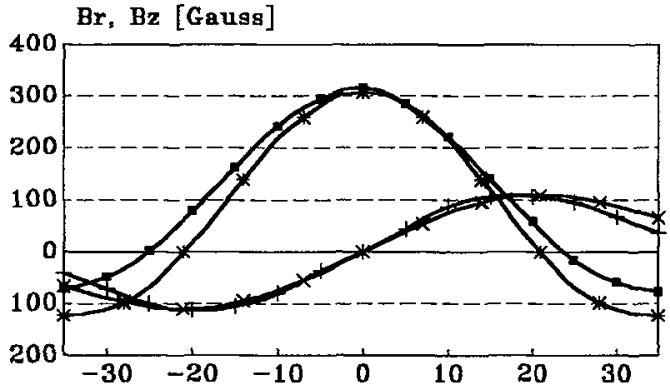

Distance from the magnetron axis [mm]

$* \operatorname{sim} . \mathrm{Br}+\exp . \mathrm{Br} * \operatorname{sim} . \mathrm{Bz}-\exp . \mathrm{Bz}$

(a)

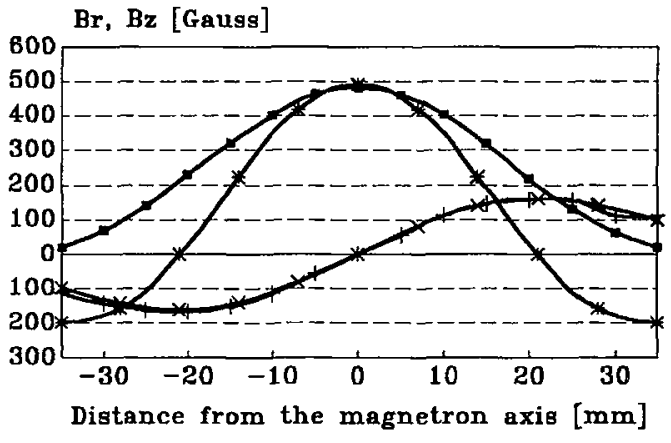

$* \operatorname{sim} . \mathrm{Br}+$ exp. Br $* \operatorname{sim} . \mathrm{Bz}-\exp . \mathrm{Bz}$

(b)

Fig. 3: The measured and simulated boundary values of the magnetic field at the substrate surface $(z=20$ $\mathrm{mm}$ ) for the different magnetron types (a): type I, (b): type II

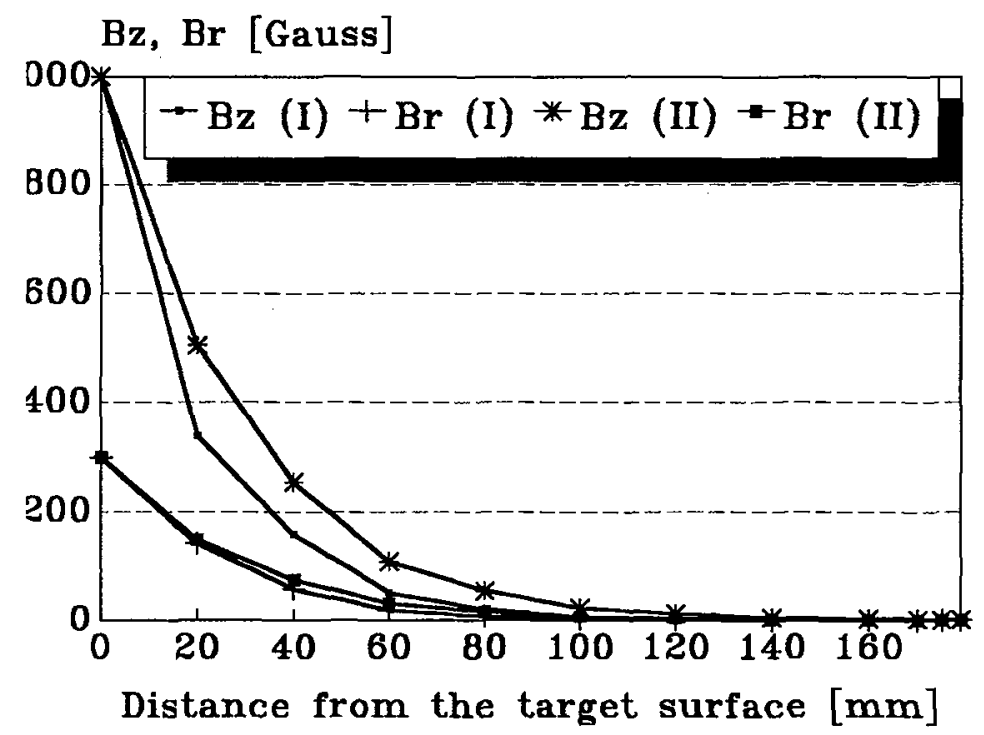

Fig. 4: The declines of the magnetic field in the process space for various magnetron types

\section{Conclusions}

Starting from the Maxwell equations one can create a simple analytic model for convenient investigation of the magnetic field in the MSIP process space which has a simple symmetry. The results show good agreement between the calculations and measurements. The analytic model will be implemented into the investigation of the motions of the charged particles in MSIP processes. 

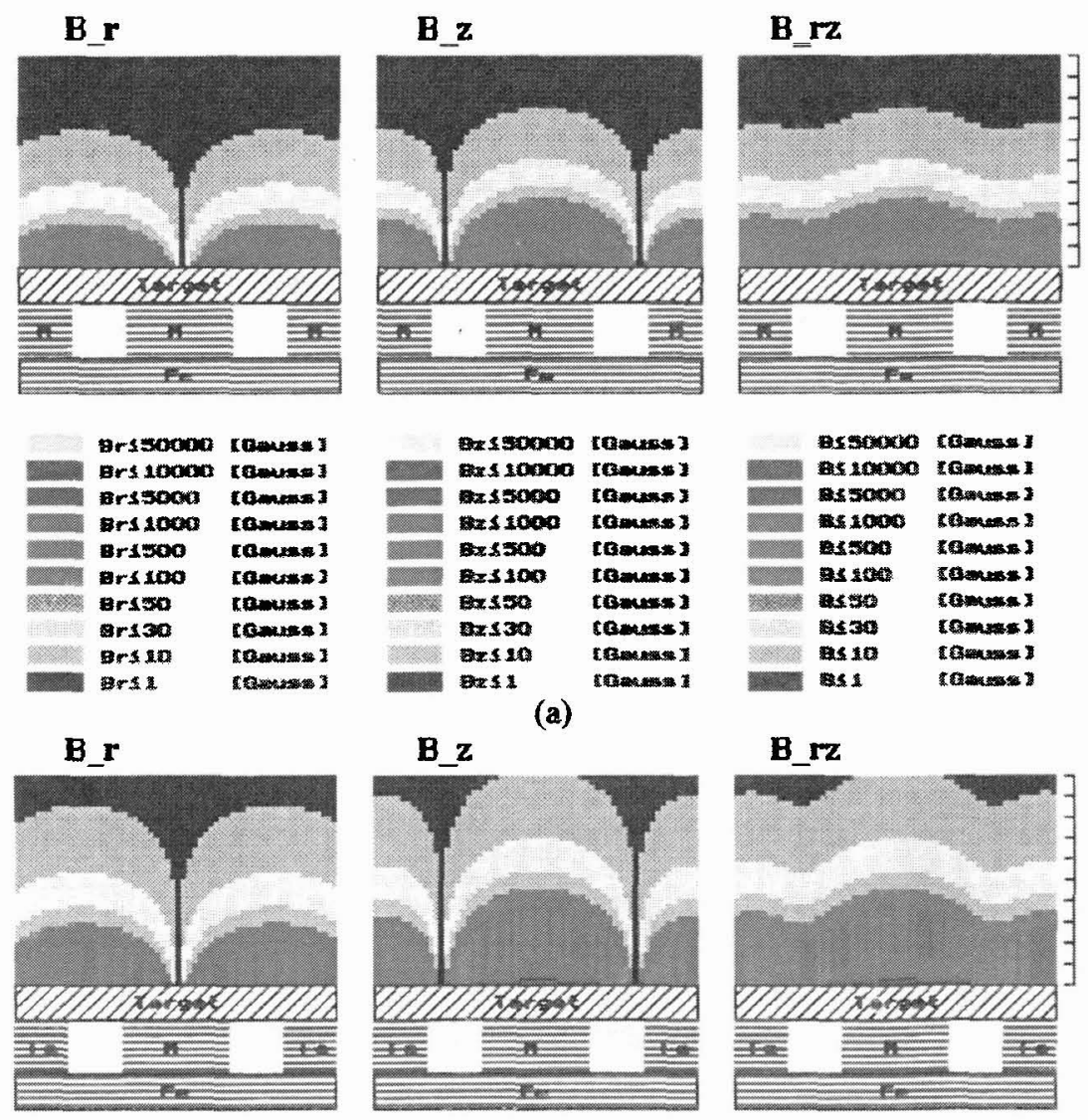

\section{B_z}
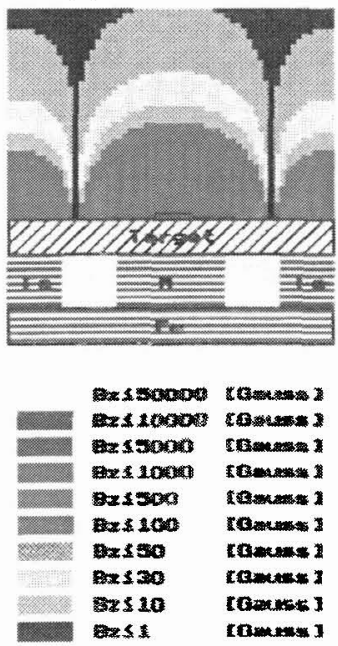
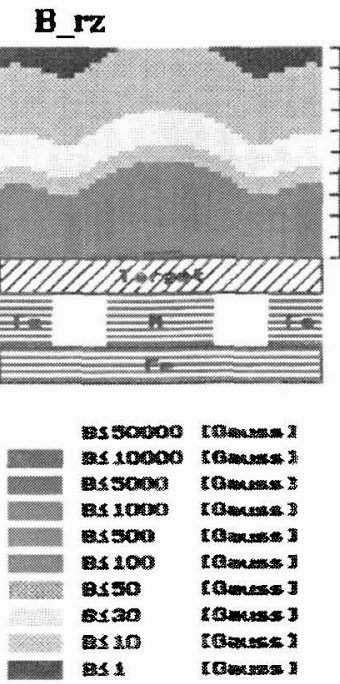

(b)

Fig.5:The distributions of magnetic field in the process space for the various magnetron types with $180 \mathrm{~mm}$ distance between the target and substrate (a): type I, (b): type II

\section{References}

11/. J.L. Vossen and W. Kern: Thin Film Processes, Academic Press, New York 1978

/2/. H. Frey und G. Kienel: Dünnschichttechnologie, Düsseldorf: VDI-Verlag 1987

13/. J.A. Thornton: Coating deposition by sputtering, in R.F. Bunshah: Deposition technologies for Films and Coatings, Noyes publications, Park Ridge, N. J., U.S.A. 1982

14/. J.D. Jackson: Classical Electrodynamics, Second edition, John Wiley \& Sons, 1975 